\title{
Enhancing Self-Awareness: A Practical Strategy to Train Culturally Responsive Social Work Students
}

\author{
Nalini J. Negi \\ Kimberly A. Bender \\ Rich Furman \\ Dawnovise N. Fowler \\ Julia Clark Prickett
}

\begin{abstract}
A primary goal of social justice educators is to engage students in a process of self-discovery, with the goal of helping them recognize their own biases, develop empathy, and become better prepared for culturally responsive practice. While social work educators are mandated with the important task of training future social workers in culturally responsive practice with diverse populations, practical strategies on how to do so are scant. This article introduces a teaching exercise, the Ethnic Roots Assignment, which has been shown qualitatively to aid students in developing self-awareness, a key component of culturally competent social work practice. Practical suggestions for classroom utilization, common challenges, and past student responses to participating in the exercise are provided. The dissemination of such a teaching exercise can increase the field's resources for addressing the important goal of cultural competence training.
\end{abstract}

Keywords: Cultural competence, cultural responsiveness, self-awareness, teaching exercise

\section{INTRODUCTION}

Social work's specialized focus on social justice requires an active engagement in finding the best strategies to address the needs of marginalized and ethnically/racially diverse populations. The importance of effectively reaching marginalized groups is especially compelling in the face of ethnic/racial minority populations' underutilization of social services (Atkinson, Morten, \& Sue, 2004). In an attempt to narrow the health disparities gap, the Council on Social Work Education's (CSWE) Educational Policy and Accreditation Standards and the Social Work Code of Ethics underscore the importance of cultural competence training in social work education. These standards require that all American social work graduates understand forms and mechanisms of oppression and discrimination and demonstrate the ability to practice with respect, knowledge, and skills related to clients' backgrounds (CSWE, 2008; NASW, 2001).

Schools of social work have undertaken the difficult task of preparing social work students to facilitate and promote culturally responsive services. Social work educators and researchers have long identified barriers to this important task, including the lack of specific operational understanding of CSWE's mandates (Horner \& Borrero, 1981;

Nalini J. Negi, Ph.D., is an Assistant Professor in the School of Social Work at the University of Maryland, Baltimore. Kimberly A. Bender, Ph.D., is an Assistant Professor at the University of Denver Graduate School of Social Work. Rich Furman, Ph.D., is Director and Associate Professor in the Social Work Program at the University of Washington, Tacoma. Dawnovise N. Fowler, Ph.D., is an Assistant Professor in the School of Social Work at the University of Texas, Austin. Julia Clark Prickett, MSW, is a student in the School of Social Work at the University of Maryland, Baltimore. 
Roberts \& Smith, 2002), and students' lack of readiness for understanding multicultural content (Lee \& Greene, 2003). In response to the complexities of training social workers for culturally competent practice, educational models such as the Newcastle model (Gibbons \& Gray, 2002) and Sakina-Mama's (2001) educational model have been developed to emphasize the value of experiential learning in social work education. However, the literature remains limited on practical teaching assignments that incorporate experiential methods to enhance students' preparation and motivation for culturally responsive social work practice.

This article outlines one such practical strategy, referred to as the Ethnic Roots Assignment, which has been used over several semesters in a graduate social justice course at a public university in the Southwest. Initial qualitative results have demonstrated the effectiveness of the assignment in engaging students in a process of self exploration and investment in culturally responsive social work practice (Bender, Negi, \& Fowler, 2010). With a focus on offering specific strategies for facilitating student selfawareness, this article describes detailed components of the Ethnic Roots Assignment, practical suggestions for implementation (including classroom utilization and navigating potential challenges), and concludes with student responses to the assignment.

\section{The Importance of Self-Awareness in Building Cultural Competency}

Social work educators, in developing teaching models for culturally competent social work practice, have emphasized the important role of self-awareness development in preparing culturally responsive social workers (Colvin-Burque, Zugazaga, \& DavisMaye, 2007; Messinger, 2004). Guided by CSWE educational principles to "engage diversity and difference in practice," social work educators encourage students to "gain sufficient self-awareness to eliminate the influence of personal biases and values in working with diverse groups" (CSWE, 2008, p. 5). Solely addressing the importance of learning about the other's culture is not sufficient as it absolves individuals from learning and understanding the impact of their own sociopolitical and ethnocentric biases on their work with clients who are racially/ethnically or culturally different from themselves. Cultural competence training must then not only encourage the continued aspiration to learn about other cultures but also aim to foster self-awareness (Green et al., 2005; Lum, 2007).

In order to prepare students for culturally responsive social work practice, social work educators have developed specific educational models to facilitate student selfreflection. For example, Sakina-Mama's (2001) educational model emphasizes the need to incorporate an understanding and acceptance of one's own culture, knowledge of other cultures, and recognition of diversity as normative. This is a paradigm that can be used as a framework for selecting readings, assignments, and facilitating classroom discussions. Gibbons and Gray's (2002) Newcastle Model similarly stresses the importance of experiential learning as instrumental in teaching cultural competence. They emphasize teaching social work students the meaning of their own personal cultural history and how to integrate this knowledge in social work practice. For example, they underscore the importance of using relevant videos, professionals in the field, and client groups to elicit critical analysis, foster student exploration and discovery, and develop intervention skills. 
The Self and Other Awareness Project (SOAP) model of instruction has also been developed as an instrument for training in cultural competence (Colvin-Burque et al., 2007). Recognizing self-awareness as a vital component of cultural competence, the SOAP model goes into more depth to identify various teaching strategies in fostering self-exploration. Through the use of large and small group activities, journal assignments, videos, guest speakers, and self-evaluations, this model has demonstrated potential to enhance student self-awareness (Colvin-Burque et al., 2007). Each of these models contributes greatly towards the development of pedagogical frameworks that allow for the selection of teaching materials that will instigate student self-awareness. However, there is a gap in literature that outlines specific teaching exercises to build self-awareness within these frameworks.

\section{THE ETHNIC ROOTS ASSIGNMENT}

One specific educational exercise that has qualitatively demonstrated promise in increasing student self-awareness regarding cultural responsiveness is the Ethnic Roots Assignment (Bender, Negi, \& Fowler, 2010). This paper assignment asks students to explore their families' ethnic roots and processes of acculturation as well as the implications of their families' experiences of their own sense of ethnic or racial identity. It also asks students to translate this renewed understanding of self to their ideas regarding cultural competence. To do so, the assignment requires students to interview their family members to gather family histories with a particular emphasis on their ancestors' processes of immigration. In addition, students are required to apply required readings regarding theories of acculturation and White-conformity to develop a critical understanding of how their family members, and, in turn, the students themselves have integrated into mainstream U.S. society (see McLemore, Romo, \& Baker, 2001). In this assignment, the process of acculturation is understood as being on a continuum influenced by families' degree of membership in the dominant culture or racial group as well as generations of immigration in the United States. For a more detailed description of the Ethnic Roots Assignment, see Appendix A. The Ethnic Roots Assignment is essentially a two-part exercise that requires students to both investigate their family history and then to write about this process in a paper. The pedagogical rationale for each section of the exercise is discussed below.

\section{Investigating Family History}

Furthering one's self-awareness is a multi-layered and dynamic process (Richardson \& Molinaro, 1996). Assigning students to interview their family members is one important way that students can begin to access their own personal history. Family interviews may give students a venue to ask their family members potentially difficult questions regarding race, ethnicity, immigration, and acculturation. The goal of this investigative exercise is to unravel patterns of privilege and discrimination that have affected their families' trajectories in the U.S.

The interview process requires students to assign meaning to facts given to them by their family members by incorporating theory about acculturation processes. This experience of eliciting information from family members will vary by student and 
provide important learning opportunities (Bender et al., 2010). Students should be able to critically examine the meaning behind what information is shared and not shared by family members. For example, for some students, family history may have been purposefully forgotten, untold, or kept a secret. This can often be an important component of the assignment and students need to be cognizant of these nuances.

\section{Writing the Ethnic Roots Paper}

The second part of the Ethnic Roots Assignment requires students to organize their experiences and thoughts, regarding the exercise of investigating their backgrounds, into a structured paper (See Appendix A). The process of investigating their own ethnic roots may be the first time many students have attempted to personalize the experience of immigration, marginalization, and acculturation. Although this process of self-discovery and self-awareness is a highly complex and mostly internal process, the paper assignment allows students to develop their thoughts more cohesively as well as provides the instructor a glimpse of this internal process. The paper is inherently descriptive but it also requires students to critically analyze these historical facts about their family through a theoretical understanding of acculturation.

\section{IMPLEMENTING THE ETHNIC ROOTS ASSIGNMENT}

\section{Classroom Utilization}

Authentic use of self. Educators using the Ethnic Roots Assignment are placed in the challenging position of creating an authentic and open atmosphere in the classroom. An introduction by the instructor at the beginning of the semester that acknowledges her/his own identity in regards to ethnic/racial background, age, gender, and experience may set the stage for students to feel comfortable exploring their own backgrounds within the classroom. Instructors must also be explicitly cognizant of the various power and racial dynamics that may exist within the classroom. By demonstrating an awareness of the role of racial dynamics, the instructor further personalizes the meaning of these dynamics as opposed to simply understanding it as a theoretical construct. For example, one co-author utilized her experience as an African American professor in a predominately White classroom as an example of how racial dynamics can play a role even within the classroom (Patton, 1999).

Drawing students' attention to their own reactions to classroom dynamics may initiate students on a path of reflection. The instructor, however, must be cautious, as the use of self requires a delicate balance between overstepping boundaries and authentic self-disclosure. The overarching goal is to create a challenging yet secure environment for discussion regarding power.

Preparation for the assignment. In addition to the educator introducing her/himself authentically, lectures preceding the Ethnic Roots Assignment should help students understand the meaning of concepts such as authenticity, identity, and genuine sense of self. Moving beyond surface-level discussions of 'treating each other with respect' and 'appreciating differences,' lectures can help students explore the psychological and social 
components of their own identity formation, thus laying a foundation for the exploration of their ethnic roots. Through such a discussion students can gain a more profound understanding of themselves as individuals with biases that must be explored in order to competently serve diverse client populations.

In preparing for the Ethnic Roots Assignment, instructors should anticipate that the intense exploration of pre-existing beliefs and biases will create anxiety for many students. This is not necessarily problematic, as instructors can help students understand their own performance issues. For the purposes of meeting the learning objectives of this assignment, instructors must present the assignment in such a way that clearly illustrates several key aims for students: 1) take ownership of their education; 2) understand the aims of the assignment; 3) be willing to grapple with an assignment for which there are no clear cut "correct answers;" 4) utilize the material for their own growth. Below is one example of how an instructor addresses these potential challenges with her students:

I challenge all of you to think about what it means to be in a social justice course about to complete an assignment about your ethnic roots. It is an opportunity for you to grow and gain new perspectives about yourself, your background, and your current viewpoints. This assignment is rumored by past students to be challenging, controversial, powerful, critical, scary, dynamic, evolving, and inspiring. Many of these rumors may hold true for you as you complete this ethnic roots assignment.

This experience has the potential to be emotionally charged. You will be challenged to think about your family's history and how that has formed who you are today. You will also have to consider the genuine thoughts, feelings, and perspectives you've developed as a result of your background. For some of you, the issues and ideas that are brought up through completing this assignment may be very emotional or feel threatening, for others it may be quite difficult to reach a depth of emotion and you may find yourself discussing ideas at a more intellectual level. It is to be expected that we will each have different reactions to exploring where we come from and how it affects us today. However, I challenge all of you to "go there"-to give yourself permission to begin this journey of thinking about how you think, and feeling what you really feel, authentically thinking about ourselves and each other and our place in society. Accepting this challenge opens the door to immense growth and the development of improved skills as future social workers.

By making such statements, the instructor can help students anticipate possible reactions to completing the assignment, recognizing that the experience will vary greatly across students. The instructor should also aim to encourage students to explore a deeper level of insight necessary for culturally competent social work practice.

After reading the assignment to students aloud, the instructor may encourage students to discuss the assignment in small groups. This allows students to normalize the struggles and challenges that they may experience as a result of the Ethnic Roots Assignment. After the assignment is discussed in small groups, students should be encouraged to ask questions or discuss any concerns. 
Debriefing the assignment after completion. Several factors should be considered when planning classroom debriefing after the completion of the assignment. Instructors should carefully consider the timing of the semester and the level of comfort developed in the classroom when deciding if students are ready to share insights gained from the assignment. A moment should be given before students turn in their assignments to discuss any residual feelings that may have risen due to the assignment's requirements. The instructor can open up the class for discussion by asking a general question such as, "Does anyone want to share before we turn in our assignments?"

If debriefing is not given careful consideration by the instructor, several problems may arise. For example, students may have concerns about evaluation and grading, especially early in the semester, which may make sharing about the assignment uncomfortable and superficial. Discussing the experience in this superficial manner can be reductionist and may diminish one of the main goals of the assignment, to elicit depth of student participation. Alternatively, discussing the assignment with too much depth may risk the potential of crossing professional boundaries as students may over-share private or sensitive information to the class and may come to regret it.

\section{Understanding and Addressing Challenges}

Student responses. While the Ethnic Roots Assignment can be helpful for many students, it should be noted that facilitating the process of self-awareness related to cultural responsiveness can be very challenging as it often involves sensitive and controversial topics (Chizhik \& Chizhik, 2005). Students may resist the level of selfreflection required to complete the Ethnic Roots Assignment. Potential sources of student resistance or apprehension include students' resistance to accepting the role of privilege in their own lives, downplaying the presence of oppression in society, and the fear of responsibility for change. For example, student resistance to exploring their racial and ethnic identity has manifested in papers that focus on a discussion of regional identity (i.e. being Texan) versus an exploration of ethnic roots and its subsequent impact on privilege or marginalization in their lives.

Furthermore, the challenge of critically analyzing oneself can be an overwhelming and an uncomfortable experience. Students have been known to respond to the Ethnic Roots Assignment with confusion, discomfort, anger, or an inability to answer the assigned questions. The assignment may be particularly challenging to those students who have difficult family relationships or may not be in communication with family members. In these cases, instructors should provide the necessary accommodations by allowing students to explore their ethnic roots in alternative ways. While a detailed exploration of an alternative assignment is outside of the scope of this article, instructors are encouraged to think through an alternative assignment for students with such difficult family backgrounds by requiring them to critically think through their ethnicity. To achieve this, students can be asked to reflect on the similarity or dissimilarity of their racial or ethnic backgrounds to their adopted or family of choice and its subsequent impact on their lives (values, ethnic identity, understanding of cultural responsiveness, among others). However, it is important that this alternative is only offered on a case by case basis and after careful examination of the student's background as instructors walk a 
fine-line between being supportive of students versus being overly caretaking. Students should be encouraged to struggle with the material enough to be challenged, but not so much that they are angry, frustrated, or disrupt class room dynamics.

Maintaining boundaries. Since the Ethnic Roots Assignment facilitates the process of self-reflection-which requires students to expose themselves emotionally-it is important that the instructor establish guidelines for appropriate disclosure early in the class. It is vital for the instructor to initiate the class with a discussion underscoring the importance of boundaries and professionalism within the classroom. Instructors should work to establish the difference between educational processes and psychotherapy. While the importance of encouraging students to engage in self-reflective processes is evident, there are also risks to which the instructor must attend. Some students find that engaging in self-reflection is a stressful and sometimes emotionally taxing process. Students with mental health concerns may be particularly vulnerable to feelings of guilt and shame over this type of self-exploration. Instructors should inform students of these potential risks by addressing these issues both in the syllabus and verbally in the beginning of the semester as well as making themselves available to discuss concerns about engaging in this type of self-reflection outside of the classroom. It is important to note that students who demonstrate serious problems maintaining boundaries should be referred to discuss their concerns with their own mental health providers.

Group dynamics. The Ethnic Roots Assignment cannot be implemented without a clear understanding of group dynamics. Every classroom of students will have their own dynamics and bring into the classroom their own backgrounds, characteristics, and experiences. The level of guardedness or open-mindedness of a few students can affect group interactions as a whole. As a class, students may work through many of the stages of group formation and these processes can affect individuals' abilities to gain insight into their own experiences. It should be expected that many students avoid disclosing their individual thoughts and experiences, while other students may more openly share their insights. Educators must be aware of the group dynamics at work and frame discussions in the context of these dynamics.

\section{STUDENT EXPERIENCES OF THE ETHNIC ROOTS ASSIGNMENT}

In an effort to explore students' processes and reactions to the assignment, the Ethnic Roots Assignment was collected and analyzed qualitatively over the course of one semester (Bender et al., 2010). Students were informed about the study and were asked for voluntary participation through submission of their Ethnic Roots Assignment paper electronically or as hard copies. All papers were de-identified before analysis. This study's protocol was approved by the Institutional Review board (IRB). The sample included the papers of 23 graduate social work students. Content analysis revealed eleven main themes emerging from the qualitative data analysis, including students' enlightenment of their privilege, experiences of cultural loss, and acknowledgement of biases and empathy as integral parts of culturally competent social work practice (detailed results are reported elsewhere in Bender et al., 2010). In general, students reported that the process of completing the Ethnic Roots Assignment challenged them to critically think through their familial history and how structural forces worked to 
privilege or marginalize their family's incorporation into mainstream American society. The findings of this study indicate that graduate social work students felt that the Ethnic Roots Assignment presented them with many eye-opening insights regarding their own history of privilege and/or marginalization in America. Student responses revealed an increased empathy for the struggles of marginalized populations and a renewed commitment to the provision of culturally responsive services. Specifically, four key themes revealed the utility of the exercise as a culturally responsive teaching exercise. First, students developed an understanding of the role of race in social work clinical practice. Particularly, White students gained perspective of their own privilege and how this may potentially translate in building a working alliance with their clients. Students expressed developing an understanding of the importance of acknowledging ethnicity and race as important variables within the therapeutic process. For example, one student wrote, "Being part of a dominant ethnic group, I have been afforded the luxury of ignorance on many issues regarding ethnicity. Through the process of exploring the history of this phenomena and my personal history further, I recognize that what was a non-culture ("White") just feels that way to me because it is the dominant culture and I am a part of it." Secondly, students recognized a need for social workers to be empathetic to the many struggles and obstacles people face when they do not belong to the dominant group. Students acknowledged that social workers, especially those of the dominant group, must begin to understand their position of privilege to be more culturally competent. One student quote reads:

By recognizing the lack of hardships I have experienced as an assimilated citizen of this country, I must consider the undeniable fact that some or many of my clients will not have had a similar experience to my own. They may not feel welcome in this country, due to legislation that tries to deny them services, curriculum that is taught in our schools, or even the way people treat them based on race, ethnicity, language, religion, dress, etc. I need to be sensitive to these differences and aware of barriers and prejudices that people from different backgrounds experience.

Thirdly, students discussed that, in order to understand clients and their backgrounds, social workers must be open and willing to learn more about their clients' worldview. Finally, students further recognized that self-awareness is an ongoing process, thus underscoring the value for continued reflection and its influence on social work practice.

While many students explicitly described the insights they gained through the process of developing self-awareness, educators using this tool should be aware that not all student growth will be so evident. One educator's perspective on the impact of this assignment follows:

The overarching purpose of the ethnic roots assignment is to foster social work graduate students' understanding of the interplay between their cultural and familial backgrounds, identity constructs, and present beliefs, values, and biases. Achieving this level of depth in self-awareness from one assignment is obviously challenging. A major part of the professor's role is facilitating this process. It is noteworthy, however, that professors often will not be privy to all of the 'light 
bulb' moments that students experience during the process nor will all of these epiphanies be unmasked in the final product of the assignment. This is to be expected and is acceptable because the depth and breadth of the process of selfunderstanding is such that significant parts may and should remain confidential to enforce the format of the educational classroom ethic. Ultimately, that which is most important, the impact on the student toward self-understanding, and the foundation of what this means for their future social work practice with clients, nonetheless has occurred.

The instructor's role, therefore, should be to guide students through each stage of the assignment, facilitating students' unique forms of progress toward self-awareness and cultural competence.

\section{CONCLUSION}

Social work educators continue to shoulder the challenge of training culturally responsive practitioners despite a dearth of published educational strategies designed to promote cultural responsiveness (Boyle \& Springer, 2001). The Ethnic Roots Assignment, with its focus on self-awareness as a vital component of cultural competence, is one such promising teaching exercise that has been demonstrated qualitatively to facilitate students' understanding and motivation to engage in culturally responsive practice. It should be noted that while this assignment shows considerable potential to increase students' self-awareness regarding cultural responsiveness, a limitation, consistent with other qualitative inquiries, is the small sample of participants included in the analysis of this exercise. Future work is then needed to quantitatively test the effects of the Ethnic Roots Assignment on student competencies and to revise the assignment to adapt to varying contexts. Furthermore, other exercises with similar goals should be shared and empirically investigated in order to broaden the field's resources for addressing the important goal of cultural competence training. As ideas and teaching techniques, such as the Ethnic Roots Assignment, are more widely disseminated across schools of social work, educators will be better equipped to employ diverse pedagogical methods in training culturally responsive social workers.

\section{References}

Atkinson, D. R., Morten, G., \& Sue, D. W. (2004). Counseling American minorities (6 $6^{\text {th }}$ ed.). Boston: McGraw-Hill.

Bender, K., Negi, N. J., \& Fowler, D. (2010). Exploring the relationship between selfawareness and future cultural competency for social work graduate students. Journal of Ethnic and Cultural Diversity in Social Work, 19(1), 34-53.

Boyle, D. P., \& Springer, A. (2001). Toward a cultural competence measure for social work with specific populations. Journal of Ethnic and Cultural Diversity in Social Work, 9(3/4), p. 53-71.

Chizhik, E. W., \& Chizhik, A. W. (2005). Are you privileged or oppressed? Students' conceptions of themselves and others. Urban Education, 40(2), 116-143. 
Colvin-Burque, A., Zugazaga, C. B., \& Davis-Maye, D. (2007). Can cultural competence be taught? Evaluating the impact of the SOAP model. Journal of Social Work Education, 43(2), 223-242.

Council on Social Work Education (CSWE). (2008). Educational Policy and Accreditation Standards. Retrieved from http://www.cswe.org/CSWE/accreditation

Gibbons, J., \& Gray, M. (2002). An integrated and experience-based approach to social work education: The Newcastle model. Journal of Social Work Education, 21 (5), 529-549.

Green, R. G., Kitson, G., Kiernan-Stern, M., Leak, S., Bailey, K., Leisey, M., et al. (2005). The multicultural counseling inventory: A measure for evaluating social work student and practitioner self-perceptions of their multicultural competencies. Journal of Social Work Education, 41(2), 191-208.

Horner, W., \& Borrero, M. (1981). A planning matrix for standard 124(A). Journal of Education for Social Work, 17, 36-43.

Lee, M. Y., \& Green, G. J. (2003). A teaching framework for transformative multicultural social work education. Journal of Ethnic and Cultural Diversity in Social Work, 12(3), 1-28.

Lum, D. (2007). Culturally competent practice: A framework for understanding diverse groups and justice issues. Belmont, CA: Brooks/Cole.

McLemore, S., Romo, H., \& Baker, S. (2001). Racial and ethnic relations in America (6 ${ }^{\text {th }}$ ed.). Boston: Allyn \& Bacon.

Messinger, L. (2004). The good, the bad, and the ugly: A teaching innovation to help students develop cultural competence. The Journal of Baccalaureate Social Work, 10(1), 61-74.

National Association of Social Workers (NASW). (2001). NASW Standards for Cultural Competence in Social Work Practice. Retrieved from http://www.socialworkers.org/sections/credentials/cultural_comp.asp

Patton, T. O. (1999). Ethnicity and gender: An examination of its impact on instructor credibility in the university classroom. Howard Journal of Communications, 10(2), 123-144.

Richardson, T. Q., \& Molinaro, K. L. (1996). White counselor self-awareness: A prerequisite for multicultural competence. Journal of Counseling and Development, 74(3), 238-242.

Roberts, T. L., \& Smith, L. A. (2002). The illusion of inclusion: An analysis of approaches to diversity within predominantly white schools of social work. Journal of Teaching in Social Work, 22(3-4), 189-211.

Sakina-Mama, R. (2001). Preparing social work students to work in culturally diverse settings. Social Work Education, 20(3), 373-382. 


\section{Author's note:}

Address correspondence to: Nalini J. Negi, Ph.D., University of Maryland School of Social Work, 525 W. Redwood Street, Baltimore, MD 21201. Email:

nnegi@ssw.umaryland.edu

\section{APPENDIX A: OUTLINE OF ETHNIC ROOTS ASSIGNMENT}

\section{Ethnic Roots Paper}

Each student will write a 5-7 page paper that explores his/her "family" ethnic roots and the unique experiences of parents, grandparents, and/or other significant caregivers in the student's life. In the paper a) describe the "family" system, b) identify the variations that arise from cultural processes, such as race and ethnicity, gender, sexual orientation, disability, socioeconomic status, and/or urban/rural differences, and c) the implications of assimilation on social and economic justice for you and your family.

The purpose of this assignment is for students to explore their own ethnic roots and examine the unique experiences of parents/grandparents/great grandparents and beyond/or other significant caregivers that have shaped their path in life. This paper will allow students to look at their roots globally as well as those prevalent in the southwestern region of the United States, if applicable.

The paper should be double-spaced, in APA format, and responding to the following set of questions. Specific information from the McLemore, Romo, and Baker (2001) reading should be incorporated and each student should personally address parts a) - d) below:

a) Background: Very briefly describe yourself (age, birthplace, social class and status when you were growing up, current cultural orientation, etc.)

b) Background of Parents/Grandparents/Great Grandparents and so on

- Description of what you know about your (1) mother, (2) father, (3) maternal grandparents, (3) paternal grandparents, (4) maternal and paternal great grandparents, and so on.

- How did ancestors enter into the United States (e.g., were they voluntary immigrants, involuntary through conquest, time of entry, etc.?).

c) Experiences with Anglo Conformity and Factors Affecting Inclusion

- By the standards of Anglo Conformity, were individuals related to you included or excluded in American society?

- How did they avoid/attempt/achieve assimilation and integration? (e.g., were names changed to fit into mainstream American society? Were ethnic roots emphasized or downplayed? Were traditions/language/customs suppressed or passed down?).

- What role did social class and social power play in their experiences? 
- Describe experiences of family members in terms of the presence or absence of (1) cultural assimilation by addition, (2) cultural assimilation by substitution, and (3) marital assimilation.

- Does the "three-generations process" of assimilation described in the reading apply to your family's experience in this country? Why or why not?

d) Conclusion: What conclusions do you personally draw about your own current status of assimilation based on your ethnic roots, socialization, and personal experiences and its implications for cultural sensitivity and culturally competent practice?

Text: McLemore, S., Romo, H., \& Baker, S. (2001). Racial and ethnic relations in America ( $6^{\text {th }}$ ed.). Boston: Allyn \& Bacon. 